\title{
The DarkSide Multiton Detector for the Direct Dark Matter Search
}

C. E. Aalseth, ${ }^{1}$ P. Agnes, ${ }^{2}$ A. Alton, ${ }^{3}$ K. Arisaka, ${ }^{4}$ D. M. Asner, ${ }^{1}$ H. O. Back, ${ }^{5}$ B. Baldin, ${ }^{6}$ K. Biery, ${ }^{6}$ G. Bonfini, ${ }^{7}$ M. Bossa, ${ }^{8}$ A. Brigatti, ${ }^{9}$ J. Brodsky, ${ }^{5}$ F. Budano, ${ }^{10}$ L. Cadonati, ${ }^{11}$ M. Cadoni, ${ }^{12}$ F. Calaprice, ${ }^{5}$ N. Canci, ${ }^{4}$ A. Candela, ${ }^{7}$ H. Cao, ${ }^{5}$ M. Cariello, ${ }^{13}$ P. Cavalcante, ${ }^{7}$ A. Chepurnov, ${ }^{14}$ A. G. Cocco, ${ }^{15}$ C. Condon, ${ }^{5}$ L. Crippa, ${ }^{9}$ D. D'Angelo, ${ }^{9}$ M. D'Incecco, ${ }^{7}$ S. Davini, ${ }^{16}$ M. De Deo, ${ }^{7}$ A. Derbin, ${ }^{17}$ A. Devoto, ${ }^{12}$ F. Di Eusanio, ${ }^{5}$ E. Edkins, ${ }^{18}$ A. Empl, ${ }^{16}$ A. Fan, ${ }^{4}$ G. Fiorillo, ${ }^{15}$ K. Fomenko, ${ }^{19}$ G. Forster, ${ }^{11}$ M. Foxe, ${ }^{1}$ D. Franco, ${ }^{2}$ F. Gabriele, ${ }^{7}$ C. Galbiati, ${ }^{5}$ A. Goretti, ${ }^{5}$ L. Grandi, ${ }^{20}$ M. Gromov, ${ }^{14}$ M. Y. Guan, ${ }^{21}$ Y. Guardincerri, ${ }^{6}$ B. Hackett, ${ }^{18}$ K. Herner, ${ }^{6}$ A. Hime,${ }^{1}$ P. Humble, ${ }^{1}$ E. Hungerford, ${ }^{16}$ Al. Ianni, ${ }^{7}$ An. Ianni, ${ }^{5}$ D. E. Jaffe, ${ }^{22}$ C. Jollet, ${ }^{23}$ K. Keeter, ${ }^{24}$ C. Kendziora, ${ }^{6}$ S. Kidner, ${ }^{25}$ V. Kobychev, ${ }^{26}$ G. Koh, ${ }^{5}$ D. Korablev, ${ }^{19}$ G. Korga, ${ }^{16}$ A. Kurlej, ${ }^{11}$ P. X. Li, ${ }^{21}$ M. Lissia, ${ }^{12}$ P. Lombardi, ${ }^{9}$ L. Ludhova, S. Luitz, ${ }^{27}$ G. Lukyachenko, ${ }^{14}$ Y. Q. Ma, ${ }^{21}$ I. Machulin, ${ }^{28}$ A. Mandarano, ${ }^{10}$ S. M. Mari, ${ }^{10}$ J. Maricic, ${ }^{18}$ L. Marini, ${ }^{10}$ D. Markov, ${ }^{28}$ J. Martoff, ${ }^{29}$ A. Meregaglia, ${ }^{23}$ E. Meroni, P. D. Meyers, ${ }^{5}$ T. Miletic, ${ }^{29}$ R. Milincic, ${ }^{18}$ M. Montuschi, ${ }^{7}$ M. E. Monzani, ${ }^{27}$ P. Mosteiro, ${ }^{5}$ B. Mount, ${ }^{24}$ V. Muratova, ${ }^{17}$ P. Musico, ${ }^{13}$ D. Montanari, ${ }^{6}$ A. Nelson, ${ }^{5}$ S. Odrowski, ${ }^{7}$ A. Odrzywolek, ${ }^{30}$ J. L. Orrell, ${ }^{1}$ M. Orsini, ${ }^{7}$ F. Ortica, ${ }^{31}$ L. Pagani, ${ }^{13}$ M. Pallavicini, ${ }^{13}$ E. Pantic, ${ }^{4,32}$ S. Parmeggiano, ${ }^{9}$ B. Parsells, ${ }^{5}$ K. Pelczar, ${ }^{30}$ N. Pelliccia, ${ }^{31}$ S. Perasso, ${ }^{2}$ L. Perasso, ${ }^{13}$ A. Pocar, ${ }^{11}$ S. Pordes, ${ }^{6}$ D. Pugachev, ${ }^{28}$ H. Qian, ${ }^{5}$ K. Randle, ${ }^{11}$ G. Ranucci, A. Razeto, ${ }^{7}$ K. Recine, ${ }^{29}$ B. Reinhold, ${ }^{18}$ A. Renshaw, ${ }^{4}$ A. Romani, ${ }^{31}$ N. Rossi, ${ }^{7}$ B. Rossi, ${ }^{5,15}$ S. D. Rountree, ${ }^{25}$ D. Sablone, ${ }^{16}$ P. Saggese, ${ }^{9}$ R. Saldanha, ${ }^{20}$ W. Sands, ${ }^{5}$ S. Sangiorgio, ${ }^{33}$ E. Segreto, ${ }^{7}$ D. Semenov, ${ }^{17}$ E. Shields, ${ }^{5}$ M. Skorokhvatov, ${ }^{28}$ M. Smallcomb, ${ }^{3}$ O. Smirnov, ${ }^{19}$ A. Sotnikov, ${ }^{19}$ Y. Suvurov, ${ }^{4}$ R. Tartaglia, ${ }^{7}$ J. Tatarowicz, ${ }^{29}$ G. Testera, ${ }^{13}$ A. Tonazzo, ${ }^{2}$ E. Unzhakov, ${ }^{17}$ R. B. Vogelaar, ${ }^{25}$ M. Wada, ${ }^{5}$ S. E. Walker, ${ }^{15}$ H. Wang, ${ }^{4}$ Y. Wang, ${ }^{21}$ A. W. Watson, ${ }^{29}$ S. Westerdale, ${ }^{5}$ R. Williams, ${ }^{1}$ M. Wojcik, ${ }^{30}$ J. Xu, ${ }^{5}$ C. G. Yang, ${ }^{21}$ J. Yoo, ${ }^{6}$ B. Yu, ${ }^{22}$ S. Zavatarelli, ${ }^{13}$ W. L. Zhong, ${ }^{21}$ and G. Zuzel ${ }^{30}$

\footnotetext{
${ }^{1}$ Pacific Northwest National Laboratory, Richland, WA 99352, USA

${ }^{2}$ APC, Université Paris Diderot, Sorbonne Paris Cité, 75205 Paris, France

${ }^{3}$ Physics and Astronomy Department, Augustana College, Sioux Falls, SD 57197, USA

${ }^{4}$ Physics and Astronomy Department, University of California, Los Angeles, CA 90095, USA

${ }^{5}$ Physics Department, Princeton University, Princeton, NJ 08544, USA

${ }^{6}$ Fermi National Accelerator Laboratory, Batavia, IL 60510, USA

${ }^{7}$ Laboratori Nazionali del Gran Sasso, 67010 Assergi, Italy

${ }^{8}$ Gran Sasso Science Institute, 67100 L'Aquila, Italy

${ }^{9}$ Physics Department, Università degli Studi and INFN, 20133 Milano, Italy

${ }^{10}$ Physics Department, Università degli Studi Roma Tre and INFN, 00146 Roma, Italy

${ }^{11}$ Physics Department, University of Massachusetts, Amherst, MA 01003, USA

${ }^{12}$ Physics Department, Università degli Studi and INFN, 09042 Cagliari, Italy

${ }^{13}$ Physics Department, Università degli Studi and INFN, 16146 Genova, Italy

${ }^{14}$ Skobeltsyn Institute of Nuclear Physics, Lomonosov Moscow State University, Moscow 119991, Russia

${ }^{15}$ Physics Department, Università degli Studi Federico II and INFN, 80126 Napoli, Italy

${ }^{16}$ Department of Physics, University of Houston, Houston, TX 77204, USA
} 


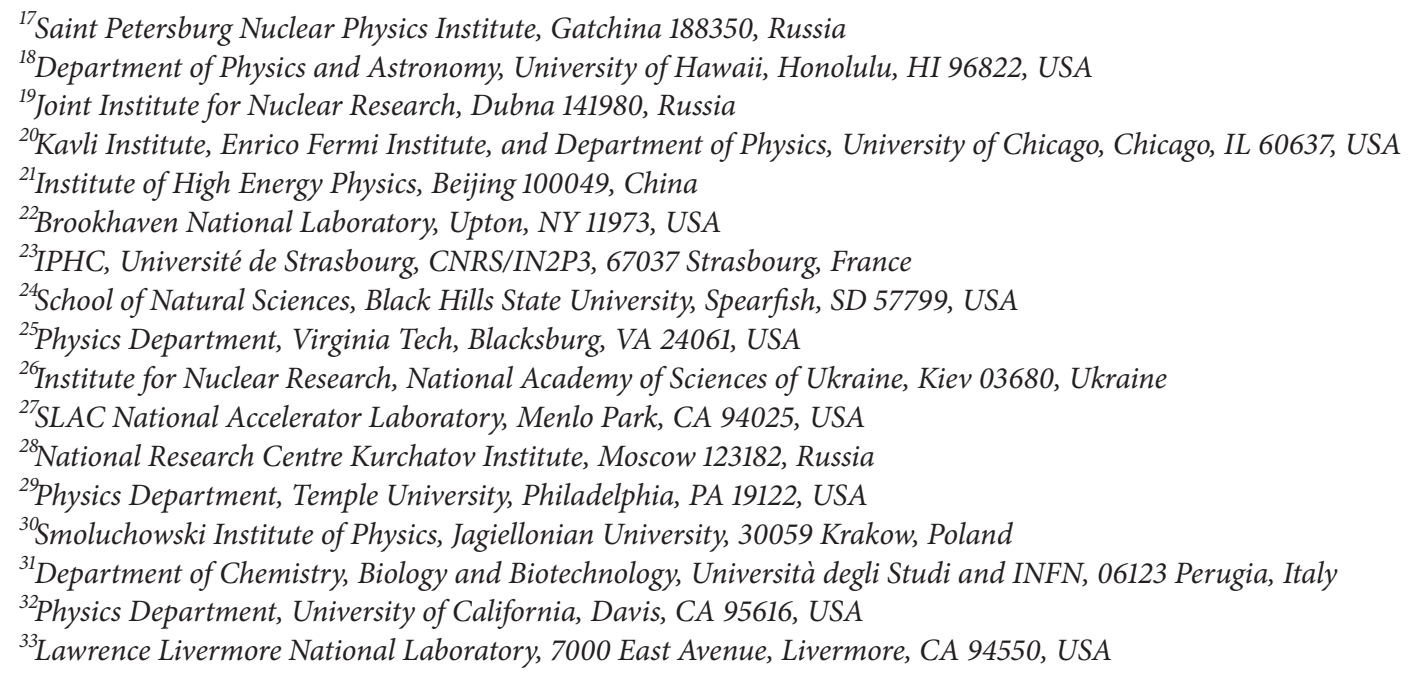

Correspondence should be addressed to G. Fiorillo; giuliana.fiorillo@na.infn.it

Received 7 March 2014; Accepted 19 June 2014

Academic Editor: Marcello Messina

Copyright (C) 2015 C. E. Aalseth et al. This is an open access article distributed under the Creative Commons Attribution License, which permits unrestricted use, distribution, and reproduction in any medium, provided the original work is properly cited. The publication of this article was funded by SCOAP S $^{3}$

Although the existence of dark matter is supported by many evidences, based on astrophysical measurements, its nature is still completely unknown. One major candidate is represented by weakly interacting massive particles (WIMPs), which could in principle be detected through their collisions with ordinary nuclei in a sensitive target, producing observable low-energy $(<100 \mathrm{keV})$ nuclear recoils. The DarkSide program aims at the WIPMs detection using a liquid argon time projection chamber (LAr-TPC). In this paper we quickly review the DarkSide program focusing in particular on the next generation experiment DarkSide-G2, a 3.6-ton LAr-TPC. The different detector components are described as well as the improvements needed to scale the detector from DarkSide50 (50 kg LAr-TPC) up to DarkSide-G2. Finally, the preliminary results on background suppression and expected sensitivity are presented.

\section{Introduction}

A wide range of astronomical evidence implies the existence of dark matter, but as yet the nature of this major component of the universe is completely unknown. A leading candidate explanation is that dark matter is composed of weakly interacting massive particles (WIMPs) formed in the early universe and gravitationally clustered together with the standard baryonic matter. Such WIMPs could in principle be detected through their collisions with ordinary nuclei in a sensitive target, producing observable low-energy $(<100 \mathrm{keV})$ nuclear recoils. The predicted collision rates are very low and require ultra-low background detectors with large (0.110 tons) target masses, located in deep underground sites to reduce the background produced by neutrons from cosmic ray muons [1-4].

Many technologies have been developed for the direct detection of dark matter WIMPs. These include cryogenic bolometers with ionization or scintillation detection (CDMS [5], EDELWEISS [6], and CRESST [7]), sodium/cesium iodide scintillation detectors (DAMA/LIBRA [8] and KIMS [9]), bubble chambers (PICASSO [10] and COUPP [11]), a point contact germanium detector (CoGeNT [12]), detectors using liquid xenon (XENON [13], ZEPLIN [14], LUX [15], and XMASS [16]), and detectors using liquid argon (miniCLEAN [17], DEAP [18], ArDM [19], and the experiment described in this paper, DarkSide). These detectors all share the common goal of achieving the low detection threshold energy required to observe the collisions of WIMPs with target nuclei and the low background to identify these extremely rare events as being from nonstandard sources.

To date, the LUX collaboration has obtained the most sensitive results for WIMPs, placing a limit of $8 \times 10^{-46} \mathrm{~cm}^{2}$ for a WIMP mass of $33 \mathrm{GeV} / \mathrm{c}^{2}$ [15]. Evidence for a low-mass dark matter signal has been claimed by the DAMA/LIBRA and CoGeNT collaborations and may be consistent with recent observations of the CDMS collaboration using $\mathrm{Si}$ detectors [20]. However, these are in apparent contradiction with the XENON [13] results and a recent low-energy analysis of CDMS-Ge detectors data [21]. They also are in strong contradiction with the recent results from LUX, which prima facie rule out the evidences for low-mass WIMPs presented by COGENT [12] and CDMS-Si [20]. The motivation for direct WIMP searches, however, remains extremely strong, especially for high $\left(>200 \mathrm{GeV} / \mathrm{c}^{2}\right)$ masses not yet explored 


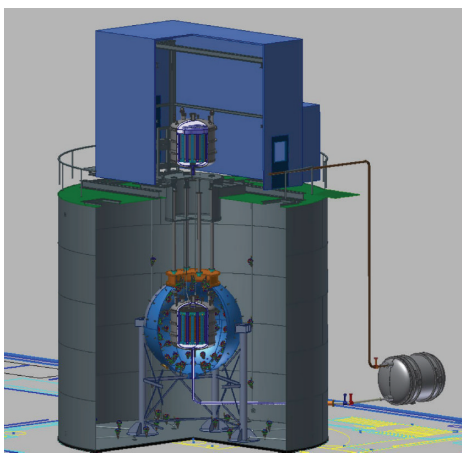

(a)

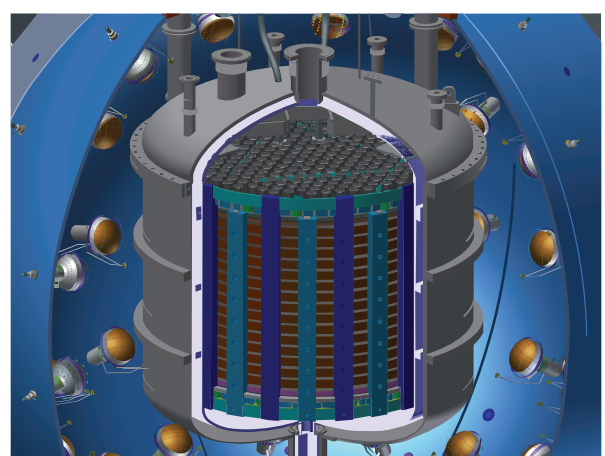

(b)

Figure 1: 3D model of the muon veto, neutron veto, and DarkSide-G2 LAr TPC (a), with a zoom on the TPC (b).

by the LHC and the region of low cross section $\left(10^{-45} \mathrm{~cm}^{2}-\right.$ $10^{-47} \mathrm{~cm}^{2}$ ) corresponding to H-mediated scattering [22].

Among the variety of detector technologies, noble liquid time projection chambers (TPCs), which detect both the scintillation light and the ionization electrons produced by recoiling nuclei, are particularly promising. Among the noble liquids, argon can effectively discriminate signal from background thanks to the time profile of the primary light signal, which depends on the interacting particle energy loss rate. In addition, liquid argon can be highly purified as demonstrated in [23] (see also references cited therein). The ability to identify, measure, and reject background makes the liquid argon TPC a key technology in dark matter searches.

The DarkSide collaboration plans to develop a multiton liquid argon TPC in the next 2-3 years. In this paper, we describe the promising results obtained with a $10 \mathrm{~kg}$ prototype, the current commissioning of an experiment with a $50 \mathrm{~kg}$ TPC, and the ongoing R\&D for DarkSide-G2, the final multiton detector.

\section{The DarkSide Experiment}

The DarkSide project is designed for direct detection of dark matter particles, at Laboratori Nazionali del Gran Sasso (LNGS), using a two-phase TPC with liquid argon (LAr) targets. The main feature of the LAr technology is the capability to efficiently separate nuclear recoil events, as expected from WIMP interactions, from electron recoil events. In addition, the high photon yield $(\sim 40000$ photons $/ \mathrm{MeV}$ for $\sim \mathrm{MeV}$ electrons at null electric field) and the optical transparency to its own light make liquid argon an ideal medium for detecting dark matter particle interactions.

As for all the direct dark matter experiments, the success of the project mostly depends on the background control, by using ultrapure materials and purification systems, identification, and suppression. The DarkSide collaboration has dedicated a notable effort in order to meet the background requirements on internal contaminations and external background from cosmic rays, detector materials, and the surrounding rocks. A particular effort has been dedicated to ${ }^{39} \mathrm{Ar}$, a $\beta$-decay with long half-life $\left(\tau_{1 / 2}=269\right.$ years and $Q$-value $=565 \mathrm{keV}$ ), intrinsic to liquid argon.
${ }^{39} \mathrm{Ar}$ is mainly formed in the atmosphere by cosmic neutron interactions on ${ }^{40} \mathrm{Ar} .{ }^{39} \mathrm{Ar}$ is, indeed, present with relatively high concentrations $\left(\sim 10^{-15}\right.$ with respect to $\left.{ }^{40} \mathrm{Ar}\right)$ in commercial liquid argon, a distillation product of liquid air. The DarkSide collaboration identified underground argon (UAr), where the exposure to cosmic rays is naturally minimized, as solution to ${ }^{39} \mathrm{Ar}$ problem. The Princeton group successfully demonstrated that ${ }^{39} \mathrm{Ar}$ is suppressed by more than 2 orders of magnitude in UAr with respect to commercial argon of atmospheric origin (AAr) [25].

A three-stage program was designed to test both the background reduction and the liquid argon technology. In particular, the staged approach allows deeply investigating the key aspect of the experiment, its background identification capabilities. The first prototype, DarkSide-10, a $10 \mathrm{~kg}$ TPC installed at LNGS inside a passive shield, provided fundamental measurements, demonstrating the high light yield achievable in a liquid argon TPC ( $~ 9$ photoelectrons/ $/ \mathrm{keV}_{e e}$ measured at null field averaging over calibration gammas from ${ }^{57} \mathrm{Co},{ }^{22} \mathrm{Na}$, and ${ }^{137} \mathrm{Cs}$ ) [23].

The current phase, DarkSide-50, already installed at LNGS, consists in $33 \mathrm{~kg}$ of liquid argon fiducial mass. It is shielded by 3500 -meter water equivalent of rock and by the double veto, a spherical borated scintillator detector, housing the TPC, designed to veto neutrons and gamma rays, and a more external water Cherenkov one to detect the residual cosmic ray flux. DarkSide-50 not only will demonstrate the background suppression efficiency but also will have the potential to provide a significant physics reach of $10^{-45} \mathrm{~cm}^{2}$ in the WIMP cross section at $100 \mathrm{GeV} / \mathrm{c}^{2}$ mass in 0.1 ton $\times$ year exposure. The data taking of DarkSide- 50 recently started and will use for the first time the UAr. This intermediate phase has already allowed the collaboration to tackle technical issues, paving the way for the following phase of the project, the so-called DarkSide-G2, a 3.6 ton TPC, with a physics goal of $10^{-47} \mathrm{~cm}^{2}$ at $100 \mathrm{GeV} / \mathrm{c}^{2}$ in 18 ton $\times$ year exposure. The double veto, commissioned for DarkSide-50, will also house DarkSide-G2, adding another brick towards the DarkSide final measurement. A schematic drawing of the muon veto, neutron veto, and LAr TPC is shown in Figure 1. 
This review is focused on the description of the third phase of DarkSide, highlighting the already achieved progress and the future needed improvements.

\section{The TPC}

The WIMP signature in a LAr TPC relies on both the excitation and the ionization of the argon. The first results in a direct scintillation signal. The latter is split into two processes: a fraction of the electron-ion pairs undergoes a recombination, which deexcites by emitting photons; the remaining free electrons are drifted towards the liquid surface, by an electric field, and are extracted into the gas phase. In the so-called gas pocket, electrons, further accelerated by a stronger electric field, excite the gas atoms producing a secondary scintillation via electroluminescence. The light components emitted in liquid argon and in gas are called $S 1$ and $S 2$, respectively.

Interacting particles can be statistically identified exploiting two features of the liquid argon response: the ratio between $S 1$ and $S 2$, which depends on the recombination probability, and the $S 1$ photoelectron time distribution, which depends on the probability of populating singlet ( $\sim 10 \mathrm{~ns}$ decay time) and triplet $(\sim 1 \mu \mathrm{s}$ decay time) states of the excited argon molecule. Both the features depend on the interacting particle stopping power. We estimate a discrimination power of $\sim 10^{2}$ for the $S 1 / S 2$ ratio and $\sim 10^{8}$ for the pulse shape discrimination $[26,27]$.

In DarkSide-G2 the dimensions of the cryostat were designed to safely accommodate the LAr-TPC, with diameter and height of $1531 \mathrm{~mm}$ and $1503 \mathrm{~mm}$, respectively, for a total active Ar mass of 3.8 ton. Taking into account the volume occupied by the TPC material and support structure and the $10 \%$ ullage fraction, the total Ar mass is 5.4 ton.

Both $S 1$ and $S 2$ signals are detected by $5563^{\prime \prime}$ Hamamatsu low-background R11065-G2 PMTs, 278 each on the top and the bottom, which view the active Ar through fused silica windows. The cylindrical surface is a reflector, coated with a wavelength shifter that absorbs the $128 \mathrm{~nm}$ scintillation photons emitted by liquid argon. The reemitted visible photons are reflected with high efficiency. The windows at the top and bottom of the cylinder are also coated with the wavelength shifter on the inner surfaces and on both surfaces with a transparent conductive layer (indium-tin-oxide (ITO)). This allows the inner window surfaces to serve as the grounded anode (top) and HV cathode (bottom) of the LAr-TPC while maintaining their outer surfaces at the average PMT photocathode potential.

The fused silica anode window has a cylindrical rim extending downward to form the diving bell holding the $1 \mathrm{~cm}$ thick gas layer of the LAr-TPC. The electron drift system consists of the ITO cathode and anode planes, a field cage, and a grid that separates the drift and electron extraction regions. A high voltage is applied between the cathode and grid to produce a vertical electric field to drift the ionization electrons upward. Outside the cylindrical wall, copper rings at graded potentials make the drift field uniform throughout the active volume. An independently adjustable potential between the grid and anode creates the fields to extract the electrons into the gas and accelerate them to create the secondary scintillation signal.

In order to build the G2 detector, however, there are still a few important points to assess. The amount of material must be as small as possible and it must have the lowest feasible radioactivity. The stainless steel for the cryostat vessel has already been identified with an acceptable radioactivity below $1 \mathrm{mBq} / \mathrm{kg}$ for ${ }^{238} \mathrm{U}$ and ${ }^{232} \mathrm{Th}$. The main concerns come from the PMTs, since at present they have a neutron yield 6 times larger than the desired limit. The solution is within reach (see Section 6.1); however, further tests are still required.

The second important item to be tackled for the G2 scaleup is the production of UAr. As mentioned in the previous section, Princeton DarkSide group demonstrated in 2007 the extraction of low radioactivity argon from underground sources of gas [25]. The actual extraction rate is $0.5 \mathrm{~kg} / \mathrm{day}$, which was largely sufficient for the DarkSide-50 detector, but the goal for the G2 phase is to reach a production rate of about $50 \mathrm{~kg} /$ day. This does not represent a major problem (see Section 6.2) but some further developments on the distillation column for final separation between $\mathrm{N}_{2}$ and UAr are needed.

A last important point related to the TPC is the choice of the electric field strength. Typically LAr TPC applies a field of about $1 \mathrm{kV} / \mathrm{cm}$ which is a compromise between a high drift velocity (about $2 \mathrm{~mm} / \mu \mathrm{s}$ ) and technical difficulties related to the feedthroughs for very high voltages. However, the recent measurements of SCENE [28] have a crucial impact on the choice of the electric field for DarkSide-G2, since they observed a significant dependence on the drift field of liquid argon scintillation from nuclear recoils of energies in the range of interest for dark matter searches. The SCENE measurement suggests that a lower drift field is important to retain the best nuclear recoil $S 1$ light yield in a TPC. We are already operating DarkSide-50 at a much lower drift field than our prototype studies, and it is likely that the optimum drift field for DarkSide-G2 will be in the range $100-200 \mathrm{~V} / \mathrm{cm}$. This choice will have important implications on the DarkSide program. On the one hand, the scale-up of the detector will be easier, relaxing the constraint coming from the HV. On the other hand, the drift velocity will be reduced to less than $1 \mathrm{~mm} / \mu$ s with a drift time of the order of 1-2 ms. This means that the requirements on the target purity from electronegative components become more challenging. We expect the requirement for the electron drift lifetime in DarkSide-G2 to be about $5 \mathrm{~ms}$.

\section{The Neutron Veto}

The DarkSide-G2 liquid scintillator neutron veto (LSV), today hosting the DarkSide-50 TPC, is a $4 \mathrm{~m}$ stainless steel sphere filled with liquid scintillator (see Figure 2 for an internal view).

The LSV is a highly efficient active veto for neutrons and gammas, and it complements the muon veto (see Section 5) as cosmogenic veto [29]. It is equipped with 110 low-background-glass-bulb 8-inch PMTs, mounted directly on the internal surface of the supporting sphere, for a $7 \%$ 


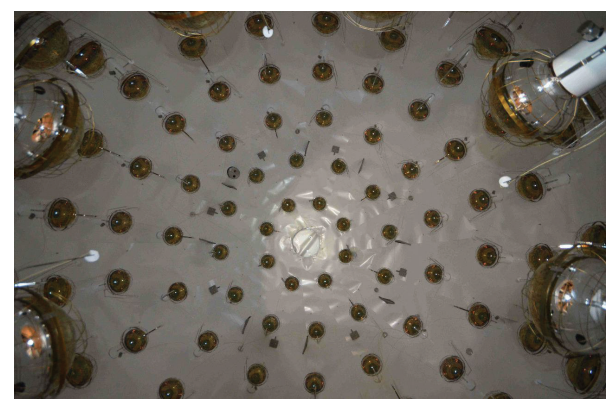

FIGURE 2: Internal view of the LSV neutron veto from its top port.

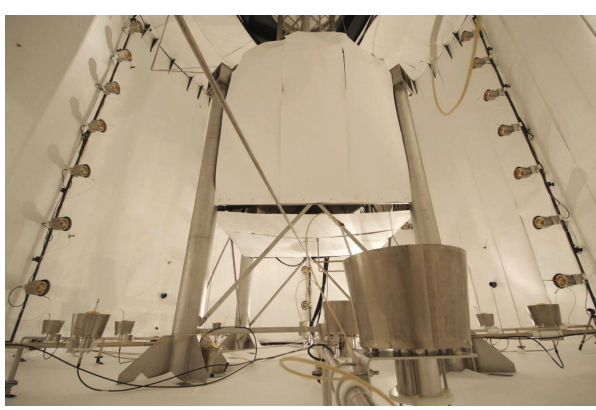

FIGURE 3: Internal view of the CTF muon veto. photocathode coverage. To maximize the light collection, a high-efficiency Lumirror reflector [30] is used to cover internally the LSV.

The scintillator is a $1: 1$ mixture of trimethyl borate (TMB) and a solution of PPO in pseudocumene (PC). Neutron capture on boron, producing $\alpha$ 's of energy $1.47 \mathrm{MeV}$ or larger, is the dominant process in the liquid, due to the large thermal neutron capture cross section on ${ }^{10} \mathrm{~B}$ and its natural abundance of $20 \%$. The fact that $\alpha$ 's are very short range particles is a critical point in order to have an efficient neutron detection without requiring very large veto volumes. This would not be the case for vetoes based on other dopants such as Gd that rely on detection of the $\gamma$-rays emitted in the neutron capture process.

The energy deposition in the scintillator due to a neutron capture on ${ }^{10} \mathrm{~B}$ is at least $60 \mathrm{keV}_{e e}[31]$, which results in a high detection efficiency. This, together with the short neutron capture time, makes it possible to operate the veto with high event rate, allowing for a direct immersion of the PMTs in the scintillator without any additional inert buffer layer.

Starting from a scintillator light yield of 12 photons $/ \mathrm{keV}_{e e}$ and typical quantum efficiency (QE) of about 35\% for the PMTs, a light yield of $0.5 \mathrm{PE} / \mathrm{keV}_{e e}$ was obtained: the resulting signal of $30 \mathrm{PE}$ for a neutron capture is more than adequate to ensure very efficient neutron detection. Last but not least, faster neutrons can also be tagged, exploiting the elastic scattering of neutrons on protons [32].

\section{The Muon Veto}

The muon and neutron vetoes were conceived in the DarkSide-50 project in order to host the G2 upgrade without any major modification. This represents an important asset for DarkSide-G2 not only in terms of cost but also in terms of detector and BG understanding.

The muon veto is the former counting test facility (CTF) $[33,34]$ of the Borexino experiment [35]. It is a Cherenkov detector $10 \mathrm{~m}$ high with a diameter of $11 \mathrm{~m}$, instrumented with 80 8-inch PMTs. It is used to identify energetic muons (and charged daughter particles) with an efficiency larger than $99 \%$ as well as acting as passive shielding for neutrons. To enhance the light collection, a reflector made of a TyvekPolyethylene-Tyvek sandwich, originally developed for the Daya-Bay reactor neutrino experiment, covers the inner surface of the tank and the external surface of the neutron veto.

An internal view of the CTF muon veto can be seen in Figure 3.

\section{Required Improvements for the Multiton Phase}

The DarkSide-50 experiment paved the way for the G2 upgrade: no modifications are needed for the muon and neutron vetoes and the TPC is almost just a scale-up by a factor of 100 .

Two points need further development, as mentioned in the previous section, namely, the photosensors and the UAr extraction.

6.1. Photosensors. The baseline for the LAr-TPC PMTs of DarkSide-G2 is the 3-inch ultra-low background Hamamatsu R11065-G2, which uses the Bialkali-LT photocathode developed by Hamamatsu Photonics under contract from INFN Napoli and Princeton University. This photocathode operates down to LAr temperature with high quantum efficiency, overcoming the limitations of standard Bialkali photocathodes which exhibit poor performance at cryogenic temperatures. Thanks to the continuing R\&D on photocathodes by Hamamatsu, in the latest models of the family, the average peak QE has risen to $37 \%$ at $400 \mathrm{~nm}$. The R11065 family of PMTs has been very successful in enabling the achievement of high photoelectron yields $\left(9 \mathrm{PE} / \mathrm{keV}_{e e}\right.$ in DarkSide-10 [23]). Nonetheless, a major concern for the $\mathrm{G} 2$ upgrade comes from the radiopurity: the very best of the family, the R11065-20, has a neutron yield of $0.6 \mathrm{n} /($ unit $\times \mathrm{yr}$ ), whereas our goal for the $\mathrm{R} 11065-\mathrm{G} 2$ is $0.1 \mathrm{n} /($ unit $\times \mathrm{yr}$ ).

This goal is within reach thanks to the identification of the leading source of radioactivity in the R11065-20 PMTs, the ceramic feedthrough plate: we measured by GDMS a localized contamination of $3 \mathrm{mBq} /$ unit ${ }^{238} \mathrm{U}$ and $1 \mathrm{mBq} / \mathrm{unit}$ ${ }^{232} \mathrm{Th}$, clustered in the ceramic feedthrough plate.

We are pursuing two routes to resolve this problem: Hamamatsu has proposed and tested a sapphire stem in the R11065-30 obtaining a reduction in background of a factor of 5 , close to the requirement of DarkSide-G2. In addition, the DarkSide collaboration has identified 99.999\% ultrahigh purity alumina, whose radiopurity will allow us to reach 


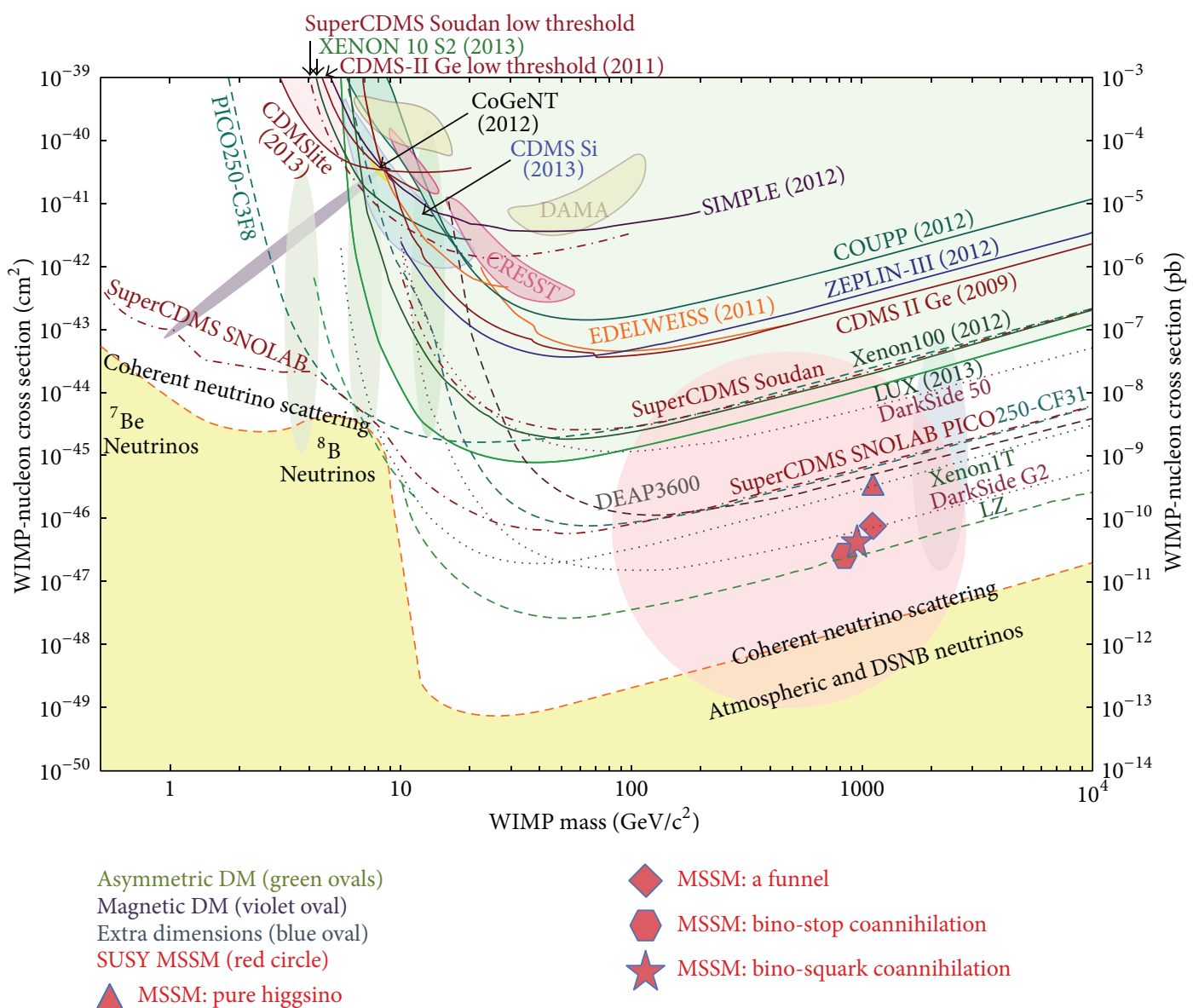

FIGURE 4: Current status of direct dark matter searches results and sensitivity of future experiments (from [24]). Shaded bounded areas represent claims for dark matter observations or regions of interest compatible with observation of a few events that could be attributed to dark matter interactions. Continuous lines show the exclusion curves by past experiments. Dashed lines show the projected sensitivity of future experiments based on various assumptions. The red dashed line shows the sensitivity of DarkSide-G2 for zero detected events in 18 tons-years.

the goal of $0.1 \mathrm{n} /($ unit $\times \mathrm{yr}$ ) and is completing $\mathrm{R} \& \mathrm{D}$ towards its use in ultraradioclean feedthroughs.

6.2. Underground Argon Distillation. To extract low radioactivity underground argon, a large vacuum-pressure swing adsorption (VPSA) plant was installed in 2009 at Kinder Morgan Doe Canyon $\mathrm{CO}_{2}$ facility in southwestern Colorado [36]. This accepts feed $\mathrm{CO}_{2}$ gas with $600 \mathrm{ppm}$ of $\mathrm{Ar}$ and produces an output stream of $4 \% \mathrm{Ar}$ in a $\mathrm{He}$ and $\mathrm{N}_{2}$ mixture. To date the VPSA plant has extracted more than $100 \mathrm{~kg}$ of UAr and it continues to extract it at a rate of about $0.5 \mathrm{~kg} /$ day.

The UAr collected in Colorado is shipped to Fermilab for further purification, using a complex purification system composed of many units, whose most important unit is a cryogenic distillation column [37]. The final obtained UAr has a contamination of ${ }^{39} \mathrm{Ar}$ lower than $0.65 \%$ compared to that in AAr [25]. At this level the DarkSide-G2 experiment will be able to operate with a very limited $(<5 \%)$ fraction of events in pile-up.

The goal for DarkSide-G2 is to reach a production of $50 \mathrm{~kg} /$ day: to meet the goal the DarkSide Collaboration is teaming with Kinder Morgan and Air Products. Air Products will make provisions to produce for the DarkSide-G2 collaboration a stream of $\mathrm{N}_{2} / \mathrm{UAr}$, originating from the tail gas of their He extraction plant, which can be treated with a cryogenic distillation column to produce detector quality UAr at the desired rate. Final separation of $\mathrm{N}_{2}$ from UAr will be performed directly at the Kinder Morgan Doe Canyon facility in Cortez by a new cryogenic distillation column based on the successful design of the unit currently operating at FNAL (the present distillation column at Fermilab has demonstrated the ability to separate a 40-60 Ar-N mix at a rate of $20 \mathrm{~kg} /$ day).

\section{Sensitivity}

In order to observe a signal rate of the order of 1 event per ton per year (assuming a cross section of $10^{-47} \mathrm{~cm}^{2}$ ), an exceptional background reduction is needed.

The sources of background are $\beta / \gamma$ giving an electron recoil misidentified as a nuclear recoil and cosmogenic or radiogenic neutrons that produce a nuclear recoil which can 
not be distinguished from a WIMP one. The strategy for an efficient background reduction relies therefore on extreme radiopurity of materials, an efficient nuclear and electron recoil discrimination, and the active external volumes to veto and suppress the neutrons reaching the TPC.

The background due to the cosmogenic neutrons can be completely eliminated using the neutron and muon vetoes. Details of the simulation studies can be found in [38], where a lifetime of 36 years was simulated and no events were found reaching the TPC active volume without triggering at least one of the two veto detectors.

The main source of radiogenic neutrons (mainly from $(\alpha, \mathrm{n})$ reactions) is the radioactivity in the PMTs and in the cryostat. Cross sections for $(\alpha, \mathrm{n})$ reactions result in one in every $10^{5}-10^{7} \quad \alpha$-decays producing a low-energy neutron [39].

Radioactive daughters of ${ }^{222} \mathrm{Rn}$ plate out on surfaces and are the major contributors to surface $\alpha$ activity. The $\alpha$-decays on the inner surface of the detector are particularly dangerous for another reason: about half the time, the $\alpha$ goes deeper into the surface, and the daughter nucleus recoils into the active volume, mimicking a WIMP recoil.

Hence, the surface contamination in DarkSide-G2 will be significantly reduced by carrying out precision cleaning and assembly in the two $\mathrm{Rn}$-suppressed clean room appositely conceived, with a Rn contamination of less than $5 \mathrm{mBq} / \mathrm{m}^{3}$.

Recoils from the remaining surface activity can be further suppressed by position reconstruction and a fiducial volume cut.

After the fiducial volume cut, the energy selection window of $55-240 \mathrm{keV}_{r}$, and the rejection of events with multiple energy depositions, we obtain less than 0.1 events from radiogenic neutrons for an exposure of 18 tons $\times$ year.

Concerning the $\beta / \gamma$ background, the main source is the ${ }^{39} \mathrm{Ar}$ in the sensitive volume, from which we expect $4.2 \times 10^{8}$ events in the dark matter energy window at the upper limit of ${ }^{39} \mathrm{Ar}$ activity for an 18-ton-year exposure. In order to achieve less than 0.1 background event in the WIMP search, the requisite reduction factor is $2 \times 10^{-10}$ between 55 and $240 \mathrm{keV}_{r}$. Pulse shape discrimination (PSD) is the major attack on this background. Preliminary data from DarkSide-50, with the same threshold, indicate that the experiment will remain background-free for the full exposure of DarkSide-G2 and with ${ }^{39} \mathrm{Ar}$ in UAr at the measured upper limit concentration [40]. Further data from DarkSide-50 are forthcoming and promise to directly test the PSD capability in LAr at the statistical level needed for DarkSide-G2.

A summary of the expected BG rate can be found in Table 1.

Based on our calculated backgrounds, the projected ( $90 \%$ C.L.) sensitivity is $2 \times 10^{-47} \mathrm{~cm}^{2}$ for $100 \mathrm{GeV}$ WIMPs, as shown in Figure 4. We note that the analysis on which we base this estimate of the sensitivity uses conservative assumptions on background rejection (DarkSide-50 data with minimal cuts and noise levels achieved at the beginning of the run, no use of $S 2 / S 1$ or of $x-y$ fiducialization yet). Possible improvements in background rejection may result in lower threshold and improved sensitivity.
TABLE 1: A summary of the expected nuclear and electron recoil backgrounds depositing 55-240 $\mathrm{keV}_{r}$ in an 18-ton-year exposure of DarkSide-G2. We assume ${ }^{39} \mathrm{Ar}$ at our recently measured limit [25]. For PMTs, we use the expected background in the R11065-G2s based on measurements reported by Hamamatsu and our ongoing R\&D. For the cryostat, we use detailed measurements of the steel used for the DarkSide-50 cryostat. After cuts include fiducial, energy, and multihit cuts.

\begin{tabular}{lcc}
\hline Detector element & $\begin{array}{c}\text { Electron recoil BG } \\
\text { after PSD and cuts }\end{array}$ & $\begin{array}{c}\text { Radiogenic neutron } \\
\text { BG after cuts }\end{array}$ \\
\hline${ }^{39} \mathrm{Ar}(<6.5 \mathrm{mBq} / \mathrm{kg})$ & $<0.1$ & - \\
R11065-G2 PMTs & $<0.01$ & 0.05 \\
Cryostat and insulation & $<0.01$ & 0.05 \\
\hline Total & $<0.1$ & 0.1 \\
\hline
\end{tabular}

\section{Conclusions}

WIMPs are a leading candidate to explain the nature of dark matter. They could be observed detecting low-energy nuclear recoils, and among the existing techniques, noble liquid TPCs are today the most promising technology.

The DarkSide program aims at the WIMPs detection using a two-phase argon TPC. The first demonstration phase of DarkSide-10 is now over and DarkSide-50 is running preliminary calibrations. The measurements taken with the two active vetoes, the borated scintillator and the water Cherenkov detectors, will allow better constraints on the background rejection efficiency of DarkSide-G2.

\section{Conflict of Interests}

The authors declare that there is no conflict of interests regarding the publication of this paper.

\section{References}

[1] Committee on the Physics of the Universe, Board on Physics and Astronomy, and Division on Engineering and Physical Sciences of the National Research Council of the National Academies, Connecting Quarks with the Cosmos: Eleven Science Questions for the New Century, National Academies Press, 2003.

[2] National Science and Technology Council, Committee on Science, A 21st Century Frontier of Discovery, The Physics of the Universe, 2004.

[3] The Dark Matter Scientific Assessment Group, Report on the Direct Detection and Study of Dark Matter, 2007.

[4] The Particle Astrophysics Scientific Assessment Group, "Report of the HEPAP Particle Astrophysics Scientific Assessment Group (PASAG)," 2009.

[5] Z. Ahmed, D. S. Akerib, S. Arrenberg et al., "Search for inelastic dark matter with the CDMS II experiment," Physical Review D, vol. 83, Article ID 112002, 2011.

[6] E. Armengaud, C. Augier, A. Beno'it [EDELWEISS Collaboration] et al., "Search for low-mass WIMPs with EDELWEISSII heat-and-ionization detectors," Physical Review D, vol. 86, Article ID 051701, 2012.

[7] G. Angloher, M. Bauer, I. Bavykina et al., "Results from $730 \mathrm{~kg}$ days of the CRESST-II Dark Matter search," European Physical Journal C, vol. 72, no. 4, pp. 1-22, 2012. 
[8] R. Bernabei, P. Belli, F. Cappella et al., "New results from DAMA/LIBRA," The European Physical Journal C, vol. 67, no. 1-2, pp. 39-49, 2010.

[9] S. C. Kim, H. Bhang, J. H. Choi et al., "New limits on interactions between weakly interacting massive particles and nucleons obtained with CsI(Tl) crystal detectors," Physical Review Letters, vol. 108, Article ID 181301, 2012.

[10] S. Archambault, F. Aubin, M. Auger et al., "Dark matter spindependent limits for WIMP interactions on 19F by PICASSO," Physics Letters, Section B: Nuclear, Elementary Particle and HighEnergy Physics, vol. 682, no. 2, pp. 185-192, 2009.

[11] E. Behnke, J. Behnke, S. J. Brice et al., "Improved limits on spindependent WIMP-proton interactions from a two liter CF3I bubble chamber," Physical Review Letters, vol. 106, no. 2, Article ID 021303, 2011.

[12] C. E. Aalseth, P. S. Barbeau, N. S. Bowden [CoGeNT Collaboration] et al., "Results from a search for light-mass dark matter with a $p$-type point contact germanium detector," Physical Review Letters, vol. 106, Article ID 131301, 2011.

[13] E. Aprile, M. Alfonsi, K. Arisaka et al., "Dark matter results from 225 live days of XENON100 data," Physical Review Letters, vol. 109, Article ID 181301, 1813.

[14] G. J. Alner, H. M. Araújo, A. Bewick et al., "First limits on WIMP nuclear recoil signals in ZEPLIN-II: a two-phase xenon detector for dark matter detection," Astroparticle Physics, vol. 28, no. 3, pp. 287-302, 2007.

[15] D. S. Akerib, X. Bai, H. M. Araujo et al., "First results from the LUX dark matter experiment at the Sanford Underground Research Facility," Physical Review Letters, vol. 112, Article ID 091303, 2014.

[16] http://www-sk.icrr.u-tokyo.ac.jp/xmass/.

[17] A. Hime, “The MiniCLEAN dark matter experiment," in Proceedings of the DPF Conference Proceedings, 2011.

[18] M. G. Boulay, "DEAP-3600 dark matter search at SNOLAB," Journal of Physics: Conference Series, vol. 375, Article ID 012027, 2012.

[19] A. Rubbia, "ArDM: a ton-scale liquid Argon experiment for direct detection of Dark Matter in the Universe," Journal of Physics: Conference Series, vol. 39, no. 39, article 129, 2006.

[20] R. Agnese, Z. Ahmed, A. J. Anderson et al., "Silicon detector dark matter results from the final exposure of CDMS II," Physical Review Letters, vol. 111, Article ID 251301, 2013.

[21] R. Agnese, A. J. Anderson, M. Asai et al., "CDMSlite: a search for low-mass WIMPs using voltage-assisted calorimetric ionization detection in the superCDMS experiment," Physical Review Letters, vol. 112, Article ID 041302, 2014.

[22] K. Zurek, "Theoretical results on Dark Matter," in Proceedings of the 26th International Symposium on Lepton Photon Interactions at High Energies, San Francisco, Calif, USA, June 2013.

[23] T. Alexander, D. Alton, K. Arisaka et al., "Light yield in DarkSide-10: a prototype two-phase argon TPC for dark matter searches," Astroparticle Physics, vol. 49, pp. 44-51, 2013.

[24] P. Cushman, C. Galbiati, D. N. McKinsey et al., "Snowmass CF1 summary: WIMP dark matter direct detection," http://arxiv.org/abs/1310.8327.

[25] J. Xu, F. Calaprice, C. Galbiati et al., "A study of the residual ${ }^{39} \mathrm{Ar}$ content in argon from underground sources," http://arxiv.org/abs/1204.6011.

[26] P. Benetti, R. Acciarrif, F. Adamo et al., "First results from a dark matter search with liquid argon at $87 \mathrm{~K}$ in the Gran Sasso underground laboratory," Astroparticle Physics, vol. 28, no. 6, pp. 495-507, 2008.
[27] M. G. Boulay and A. Hime, "Technique for direct detection of weakly interacting massive particles using scintillation time discrimination in liquid argon," Astroparticle Physics, vol. 25, pp. 179-182, 2006.

[28] T. Alexander, H. O. Back, H. Cao et al., "Observation of the dependence on drift field of scintillation from nuclear recoils in liquid argon," Physical Review D, vol. 88, Article ID 092006, 2013.

[29] A. Wright, P. Mosteiro, B. Loer, and F. Calaprice, "A highly efficient neutron veto for dark matter experiments," Nuclear Instruments and Methods in Physics Research A: Accelerators, Spectrometers, Detectors and Associated Equipment, vol. 644, no. 1, pp. 18-26, 2011.

[30] http://www.toray.us/products/electronics/ele_003.html.

[31] R. Greenwood and N. R. Chellew, "Improved ${ }^{10}$ B-loaded liquid scintillator with pulse-shape discrimination," Review of Scientific Instruments, vol. 50, p. 466, 1979.

[32] M. Flaska and S. A. Pozzi, "Digital pulse shape analysis for the capture-gated liquid scintillator BC-523A," Nuclear Instruments and Methods in Physics Research A: Accelerators, Spectrometers, Detectors and Associated Equipment, vol. 599, no. 2-3, pp. 221225, 2009.

[33] G. Alimonti, G. Anghloherd, C. Arpesella et al., "Ultra-low background measurements in a large volume underground detector," Astroparticle Physics, vol. 8, no. 3, pp. 141-157, 1998.

[34] G. Alimonti, C. Arpesella, G. Bacchiocchi et al., "A large-scale low-background liquid scintillation detector: the counting test facility at Gran Sasso," Nuclear Instruments and Methods in Physics Research A: Accelerators, Spectrometers, Detectors and Associated Equipment, vol. 406, no. 3, pp. 411-426, 1998.

[35] G. Alimonti, C. Arpesella, H. Back et al., “The Borexino detector at the Laboratori Nazionali del Gran Sasso," Nuclear Instruments and Methods in Physics Research A, vol. 600, no. 3, pp. 568-593, 2009.

[36] H. O. Back, F. Calaprice, C. Condon et al., "First large scale production of low radioactivity argon from underground sources," http://arxiv.org/abs/1204.6024.

[37] H. O. Back, T. Alexander, A. Alton et al., "First commissioning of a cryogenic distillation column for low radioactivity underground argon," http://arxiv.org/abs/1204.6061.

[38] A. Empl, R. Jasim, E. Hungerford, and P. Mosteiro, "Study of cosmogenic neutron backgrounds at LNGS," Submitted, http://arxiv.org/abs/1210.2708.

[39] R. Heaton, H. Lee, P. Skensved, and B. C. Robertson, "Neutron production from thick-target $(\alpha, \mathrm{n})$ reactions," Nuclear Instruments and Methods in Physics Research A, vol. 276, no. 3, pp. 529-538, 1989.

[40] R. Agnese, T. Alexander, A. Alto et al., "First results from the darkside-50 dark matter experiment at Laboratori Nazionali del Gran Sasso," http://arxiv.org/abs/1410.0653. 

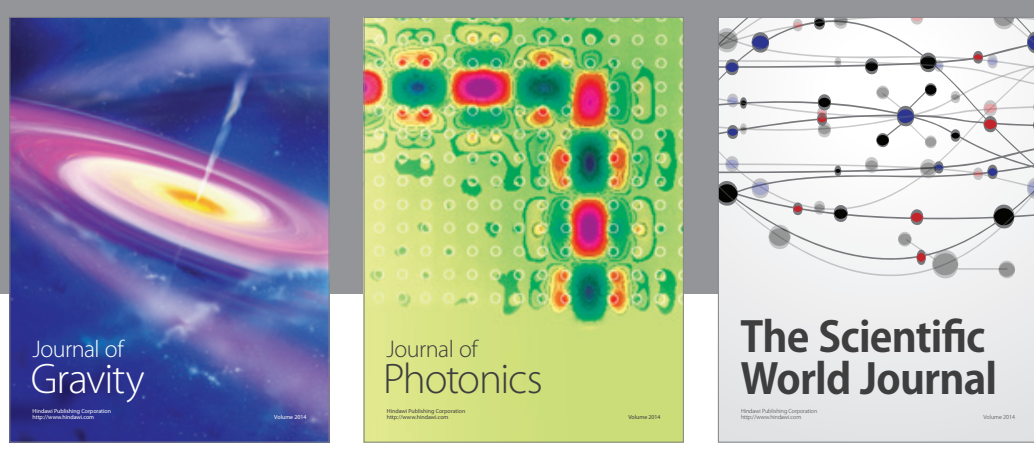

The Scientific World Journal
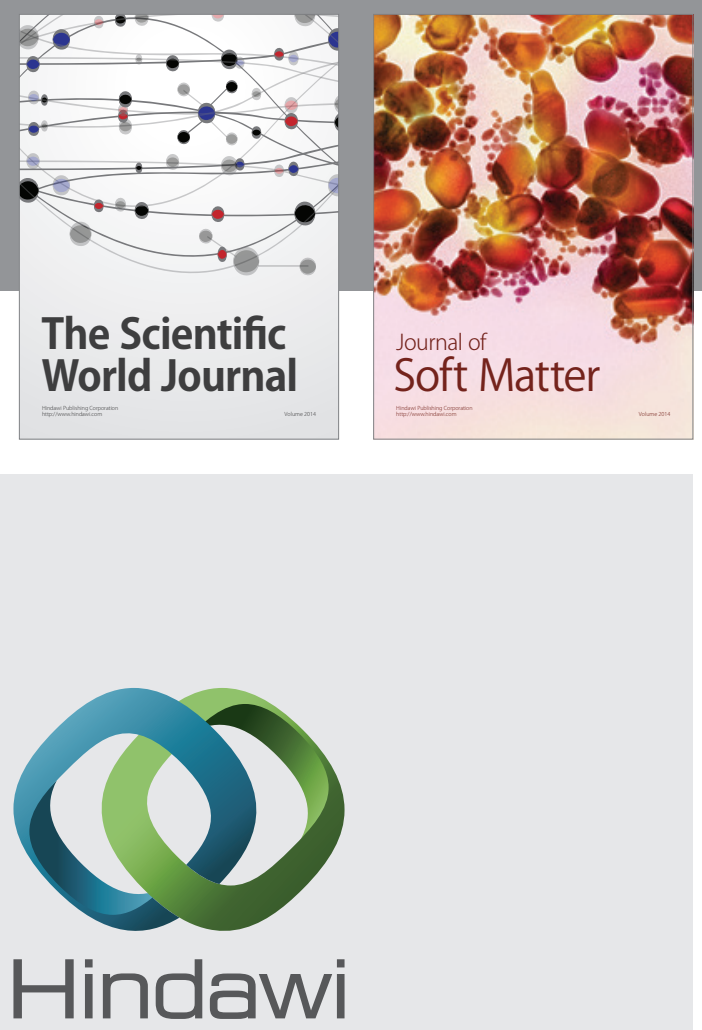

Submit your manuscripts at

http://www.hindawi.com

nternational Journal of

Statistical Mechanics
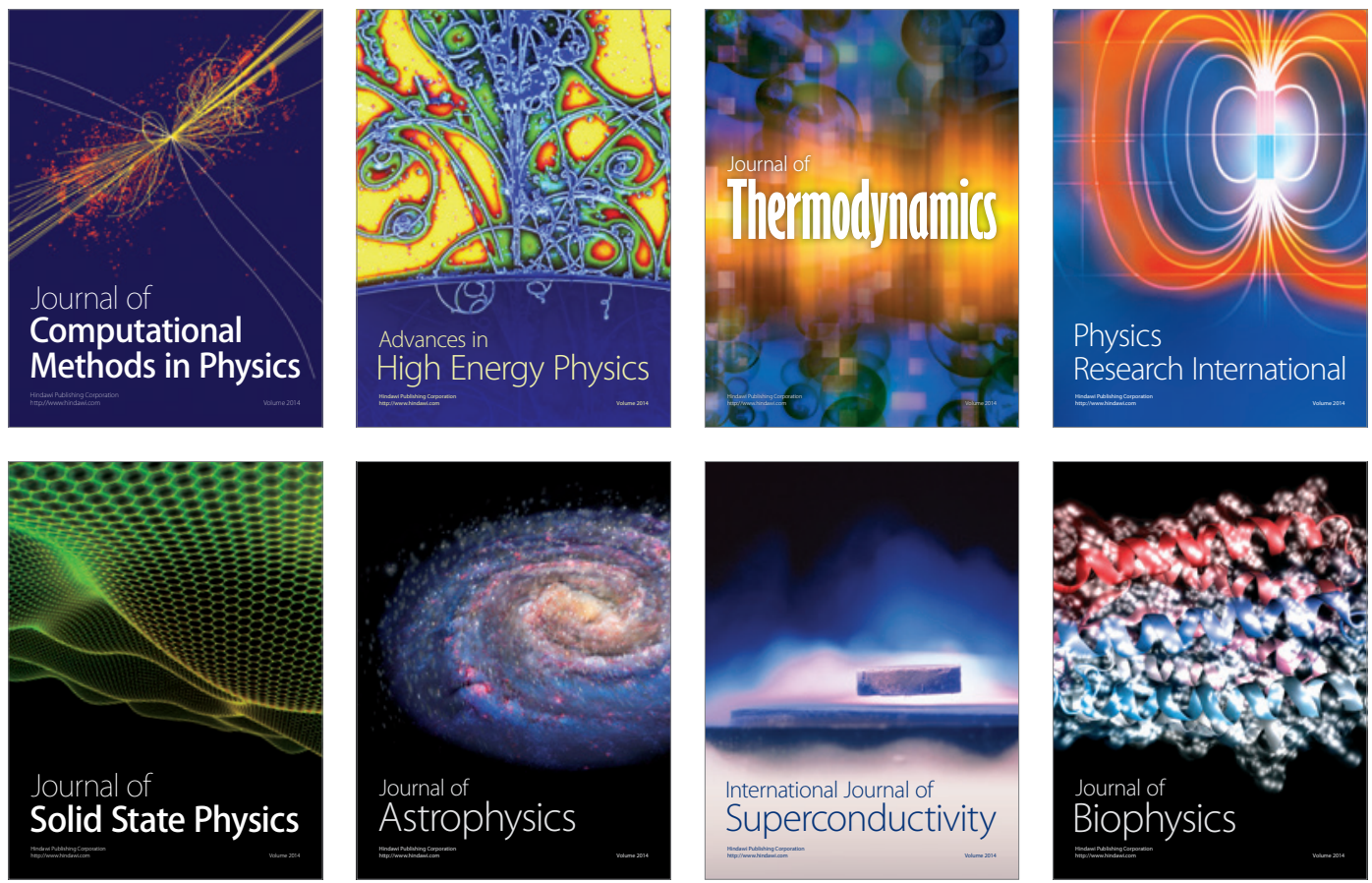
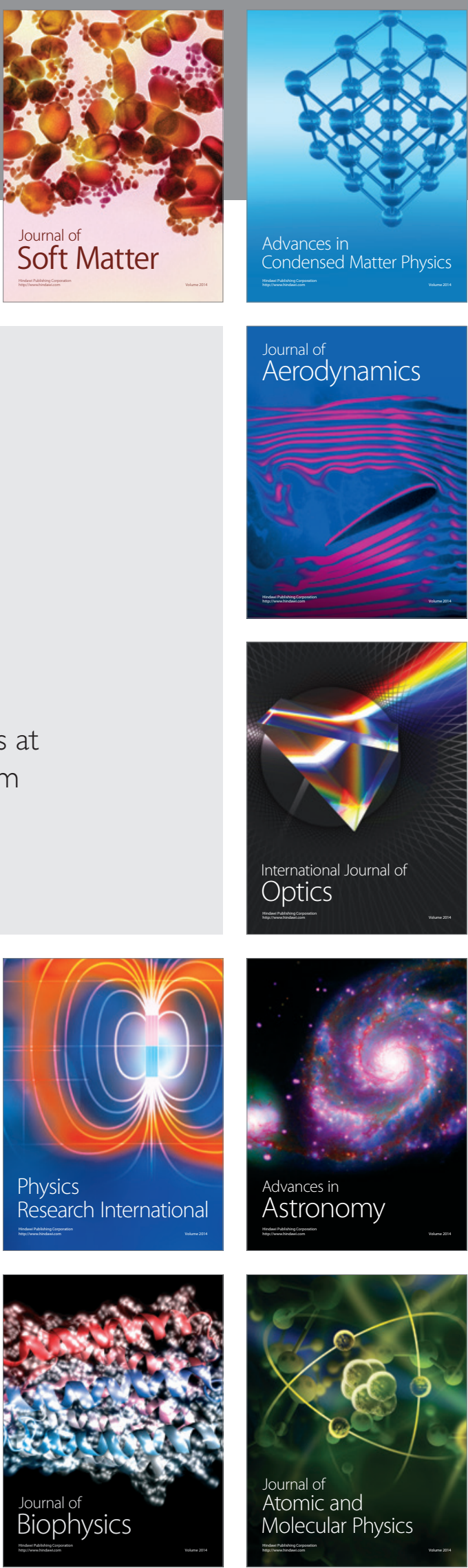